\title{
Animal memory: The contribution of generalization decrement to delayed conditional discrimination retention functions
}

\author{
RebecCA RAYburn-ReEves and Thomas R. ZENTALL \\ University of Kentucky, Lexington, Kentucky
}

\begin{abstract}
Delayed conditional discriminations in which a sample indicates which comparison stimulus is correct have typically been used in working memory research with animals. Following acquisition with no (0-sec) delay between the offset of the sample and the onset of the comparison stimuli, delays of variable duration are introduced. The resulting retention functions are taken as a measure of memory. We suggest that, in addition to memory loss due to the delay, the comparison of matching accuracy at the 0 -sec training delay with relatively novel test delays may produce a generalization decrement that varies as a function of increasing delay. We tested this hypothesis by training pigeons with a mixed delay procedure from the start and found that the retention functions for these pigeons were significantly shallower than those for a control group trained with 0 -sec delays and tested with longer delays, and, although reduced in magnitude, the differences persisted for as many as 15 sessions. We propose that a measure of animals' working memory can be obtained uninfluenced by a generalization decrement if they have received comparable training with all of the delays that are tested.
\end{abstract}

In an effort to identify basic properties of working memory separate from language and culture (e.g., learned strategies such as mnemonics), researchers have often turned to research with animals. The ease with which pigeons acquire conditional discriminations (such as matching to sample) has made them a favored species with which to study working memory (see Roberts, 1998).

The standard method for studying working memory with pigeons has been to train them on a conditional discrimination in which each of two samples indicates which of two comparison stimuli should be chosen for the pigeon to obtain reinforcement. Typically, the comparison stimuli appear immediately following the offset of the sample (0-sec delay). Once matching accuracy reaches a high level, a delay that varies in duration from trial to trial is inserted between the offset of the sample and the onset of the comparison stimuli, allowing one to plot a retention function of matching accuracy as a function of delay (Blough, 1959; Clement \& Zentall, 2000; Grant, 1975, 1981; Grant \& Roberts, 1973; Roberts, 1972, 1974; Santi \& Hope, 2001; Santi, Lellwitz, \& Gagne, 2006; Singer, Klein, \& Zentall, 2006; Zentall, Hogan, Howard, \& Moore, 1978; see also a similar procedure in delayed matching research with monkeys in Eacott, Gaffan, \& Murray, 1994). One reason that investigators have typically trained with a 0 -sec delay prior to testing with longer delays may be, as Berryman, Cumming, and Nevin (1963) reported, that pigeons exposed to delays from the start of matching training failed to acquire the matching task. However, they trained their pigeons for only nine sessions, and some of their delays were quite long $(1,2,4,10$, and $24 \mathrm{sec})$.

There has been little challenge to the basic methodology involving training with no delay and introducing delays following acquisition, which has remained in use for almost 50 years (see Berryman et al., 1963). The problem with the basic methodology is that the novelty of the delays may result in a decrement in matching accuracy (i.e., a generalization decrement) that cannot be attributed to sample forgetting (see Zentall, 1997). The fact that pigeons often perform the conditional discrimination at levels close to chance $(50 \%)$ with delays as short as 5-10 sec (Berryman et al., 1963; Grant \& Roberts, 1973; Zentall \& Hogan, 1977) suggests the possibility that such retention functions may not accurately represent the pigeon's ability to remember the sample.

In fact, Sargisson and White (2001) have shown that when pigeons are trained with a nonzero delay, their matching accuracy is poorer not only when the delay is increased but also when it is decreased. Similarly, Dorrance, Kaiser, and Zentall (2000) have shown that when pigeons are familiar with the delays used in testing, matching accuracy is better than when they are not (Sherburne, Zentall, \& Kaiser, 1998; see also Spetch \& Rusak, 1989). These results suggest that the typical retention function may consist of two components: a loss of matching accuracy resulting from the delay (or a loss in sample discriminability) and a generalization decrement related to the difference in delay between training and testing (see White, 2001). 
Occasionally, pigeons have been tested for extended periods of time, but even then, the experience with the delays used in testing has not been equated (e.g., White, 1985; but see DeLong \& Wasserman, 1981, who used delayed successive matching, a somewhat different task), and appreciation of the potential role of the generalization decrement has not been acknowledged. With the exception of White (2001; Sargisson \& White, 2001), none of these prior researchers has acknowledged that differential experience with the delays may have an effect on the slope of the retention function.

The purpose of the present study was to ask whether training with delays might eliminate the effect of the generalization decrement and might produce an unconfounded measure of pigeon working memory. The experiment involved two groups: an experimental group that was trained with the delays used during testing and a control group that was trained with the more conventional 0 -sec delay and tested with longer delays.

A second purpose of the present experiment was to ask whether extending the delays beyond those used in training would result in a new generalization decrement (a discontinuous decrement in matching accuracy not easily attributable to memory loss alone). Alternatively, experience with delays may alert the pigeons to the variability of the time between the offset of the sample and the onset of the comparison stimuli, resulting in a relatively continuous decrement in matching accuracy suggestive of memory loss alone. That is, experience with delays may generalize to longer delays.

\section{METHOD}

\section{Subjects}

Eight White Carneaux pigeons (Columba livia), ranging in age from 2 to 9 years, served as subjects. They had all served in a previous, unrelated study involving simple simultaneous discriminations without delays. Throughout the experiment, the pigeons were maintained at $80 \%-85 \%$ of their free-feeding weights. They were individually housed in wire cages, with free access to water and grit, in a colony room maintained on a 12:12-h light:dark cycle. The pigeons were maintained in accordance with a protocol approved by the Institutional Animal Care and Use Committee at the University of Kentucky.

\section{Apparatus}

The experiment was conducted in a BRS/LVE (Laurel, MD) sound-attenuating standard operant test chamber measuring $35 \mathrm{~cm}$ high, $30 \mathrm{~cm}$ from the response panel to the back wall, and $35 \mathrm{~cm}$ across the response panel. Three circular response keys $(2.5 \mathrm{~cm}$ in diameter) were aligned horizontally on the response panel and were separated from each other by $6.0 \mathrm{~cm}$. The bottom edge of the response keys was $24 \mathrm{~cm}$ from the wire-mesh floor. A 12-stimulus in-line projector (Industrial Electronics Engineering, Van Nuys, CA) with 28-V, 0.1-A lamps (GE 1820) that projected red and green hues (Kodak Wratten Filter Nos. 26 and 60, respectively) was mounted behind each response key. Mixed-grain reinforcement (Purina Pro Grains - a mixture of corn, wheat, peas, kafir, and vetch) was provided from a raised and illuminated grain feeder located behind a horizontally centered $5.1 \times 5.7 \mathrm{~cm}$ aperture and vertically midway between the response keys and the floor of the chamber. Reinforcement consisted of $1.5 \mathrm{sec}$ of access to mixed grain. A houselight (GE 1820), which provided general illumination between trials, was mounted $5 \mathrm{~cm}$ above the center response key. The experiment was controlled by a microcomputer located in an adjacent room.

\section{Procedure}

Pretraining. Each subject was pretrained to peck at both red and green stimuli on all three response keys. An increasing response requirement was required on the center key, which served as the sample, until each subject pecked 10 times to receive reinforcement. A single peck on an illuminated side key produced reinforcement.

General training. All of the pigeons were trained on matching to sample with red and green samples and red and green comparison stimuli. Each trial began with the onset of one of the two samples. A 10-peck observing response was required to each sample to turn off the sample and to turn on the red and green comparison stimuli on the left and right comparison keys. A single peck to the comparison stimulus that matched the sample was reinforced with $1.5 \mathrm{sec}$ of access to grain and began a 10 -sec intertrial interval, during which the houselight was lit. Each session consisted of 96 trials, with an equal number of red and green samples and an equal number of matching comparisons on the left and right keys randomly presented (with the constraint that a trial type was not presented more than two times successively).

Control group: Training with $\mathbf{0}$-sec delay. Four pigeons were randomly assigned to the control group and were trained on matching to sample with a 0 -sec delay between sample offset and comparison onset. The pigeons in the control group were trained to a criterion of $87.5 \%$ correct ( 84 correct matches out of 96 trials per session) or higher matching accuracy for two consecutive sessions.

Experimental group: Training with delays. The remaining 4 pigeons were assigned to the experimental group and were trained from the start on matching to sample with dark delays of $0,1,2$, or $4 \mathrm{sec}$ between sample offset and comparison onset. An equal number of the 16 trial types $(2$ samples $\times 2$ locations for the correct comparison $\times 4$ delays) occurred with each delay during each session. The pigeons in the experimental group were trained until they attained a criterion of $87.5 \%$ correct ( 21 correct matches out of 240 -sec delay trials per session) or higher matching accuracy on 0 -sec delay trials for two consecutive sessions. The training criterion for each group approximately equated for 0 -sec delay matching accuracy.

Delay Set 1. Once each bird reached the training criterion, it was tested with delays of $0,1,2$, and $4 \mathrm{sec}$ between sample offset and comparison onset. The delays were dark. Each of the four delays occurred equally often during each session and were equally distributed among the four training trial types. For the pigeons in the experimental group, the testing sessions were an extension of their training sessions. There were 96 trials in each testing session. All of the pigeons were tested for five sessions.

Delay Set 2. On the session immediately following the last session of testing with Delay Set 1 , five additional testing sessions were conducted with delays of $0,2,4$, and $8 \mathrm{sec}$ between sample offset and comparison onset.

Delay Set 3. On the session immediately following the last session of testing with Delay Set 2, five additional testing sessions were conducted with delays of $0,4,8$, and 16 sec between sample offset and comparison onset.

\section{RESULTS}

The pigeons in both groups readily acquired the delayed conditional discrimination. The pigeons in the control group, trained on 0 -sec delayed matching, acquired the task to a criterion of 2 sessions at $87.5 \%$ correct or better in an average of 10.0 sessions $(S E M= \pm 1.08)$. The pigeons in the experimental group, trained on mixed delays of $0,1,2$, and $4 \mathrm{sec}$, acquired 0 -sec delayed matching in an average of 11.0 sessions $(S E M= \pm 2.55)$. Although the pigeons in 
the experimental group had only $25 \%$ as many 0 -sec delay trials per session as did the pigeons in the control group, the difference in sessions to criterion for 0 -sec delayed matching between the experimental and control groups did not reach statistical significance [two-tailed $t(6)<1$ ] .

\section{Delay Set 1}

Matching accuracy as a function of delay was generally better for the experimental group than for the control group. A mixed model ANOVA, with group and delay as factors, performed on the first session of Delay Test 1, indicated that the effect of delay was significant $[F(3,18)=$ $28.86, p<.01]$, but the effect of group was only marginally significant $[F(1,6)=5.05, p=.066]$. More important for the present hypothesis, the group $\times$ delay interaction was statistically reliable $[F(3,18)=3.23, p=.047]$, suggesting that the retention function for the control group had a significantly steeper slope than the retention function for the experimental group. The data from Delay Set 1 on Session 1 appear in Figure 1A. At the 0-sec delay, the difference in matching accuracy between the experiment and control groups was not statistically reliable $[t(6)<1]$. Although the difference in matching accuracy between the experiment and the control groups was not statistically reliable at the $0-, 1-$, and 2 -sec delays $[t(6)<1, t(6)=1.06$, and $t(6)=1.66$, respectively]; it was statistically reliable at the 4-sec delay $[t(6)=4.12, p=.009]$.

Presented in Figures 1B-1F are the data from each of the other four test sessions and the combined data from all five test sessions. Although none of the effects of group or the interaction of group with delay was statistically reliable (largely due to the small number of subjects in each group and to considerable variability), there is no evidence that the effect actually dissipates (see the data from all five sessions combined in Figure 1F).

\section{Delay Set 2}

A mixed model ANOVA, with group and delay as factors, performed on the pooled data from the five sessions of Delay Test 2, indicated that the effect of delay was significant $[F(3,18)=24.42, p<.01]$, and although there appeared to be a small residual benefit to the group trained with delays, neither the effect of group nor the delay $\times$ group interaction was statistically significant (both $F_{\mathrm{S}}<1$ ). The data from Delay Set 2 appear in Figure 2. The difference in matching accuracy between the experiment and the control groups was not statistically reliable at any of the delays (all $t \mathrm{~s}<1$ ).

\section{Delay Set 3}

A mixed model ANOVA, with group and delay as factors, performed on the pooled data from the five sessions of Delay Test 3, indicated that the effect of delay was significant $[F(3,18)=31.85, p=.0001]$, and although there again appeared to be a residual effect of prior training with delays, neither the effect of group $[F(1,6)=3.57, p=$ .11] nor the delay $\times$ group interaction $[F(3,18)=2.50$, $p=.09]$ was statistically significant. The difference in matching accuracy between the two groups was statisti- cally reliable at the 1 -sec delay $[t(6)=3.57, p=.012$, two-tailed], but not at the 4-sec delay [ $t(6)=1.42, p=$ $.21]$, the 8 -sec delay $(t<1)$, or the 16 -sec delay $(t<1)$. The absence of an effect of prior training with delays may have been caused by a performance floor at the longer delays, especially at the 8- and 16-sec delays. The data from Delay Set 2 appear in Figure 3.

\section{DISCUSSION}

The results of the present experiment indicate that when pigeons are trained with delays, they show less forgetting than with the more typical procedure in which they are trained without delays and tested with delays, particularly on the first test session. The implication of this finding is that the typical procedure for assessing retention functions overestimates the amount of forgetting, because it includes a generalization decrement unrelated to the animal's loss of memory (White, 2001). Consistent with the hypothesis that at least some of the loss of matching accuracy that accompanies the introduction of delays can be attributed to a generalization decrement, pigeons generally show improved matching accuracy with added experience with delays (e.g., Berryman et al., 1963; Grant, 1976; Roberts \& Grant, 1976).

A more cognitive account of the difference in matching accuracy between the two groups is that pigeons that have had experience with delays during training may be more effective at remembering or may have developed better memory strategies. However, in the absence of more specific predictions concerning how such strategies might affect delayed matching performances, it is more parsimonious to propose that the generalization decrement is the mechanism responsible for the group differences found.

Although the difference in retention functions between the two groups was statistically reliable only on the first session of testing at the longest delays of the shortest delay set, the magnitude of the effect was similar over the 5 days of testing. Furthermore, with the longer delay sets, although the group difference was relatively small on Delay Set 2, it appeared to recover a bit on Delay Set 3 . Thus, although somewhat variable, group differences in matching accuracy persisted over the 15 test sessions of the present experiment.

The results of the present experiment support the results of earlier research, which suggest that training with delays produces flatter retention functions than training with a 0 -sec delay and testing with longer delays. Using white-light samples of 2 and $10 \mathrm{sec}$, Sherburne et al. (1998) trained pigeons with a 0 -sec delay to a criterion of $90 \%$ correct matching responses and tested them with delays of 0 , 1,2 , and $4 \mathrm{sec}$ for 12 sessions. The pigeons averaged $85.3 \%$ correct matching responses at the 0 -sec delay and declined to $64.5 \%$ correct at the 4 -sec delay, a difference of $20.8 \%$. However, when Dorrance et al. (2000) trained pigeons with $0-, 1-, 2-$, and 4-sec delays using a similar sample discrimination and, after they had attained a criterion of $75 \%$ correct at the 0 -sec delay, tested them for 15 sessions, they found that the pigeons averaged $87.9 \%$ correct matching 
A

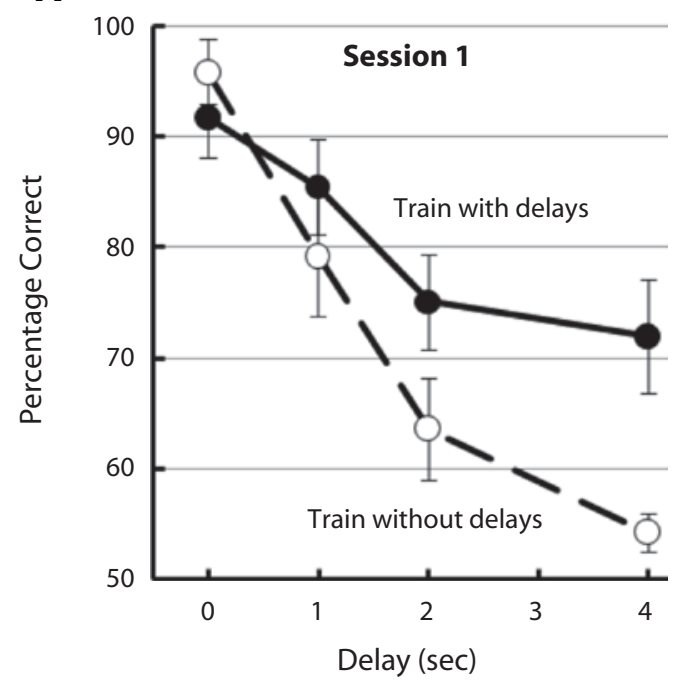

C

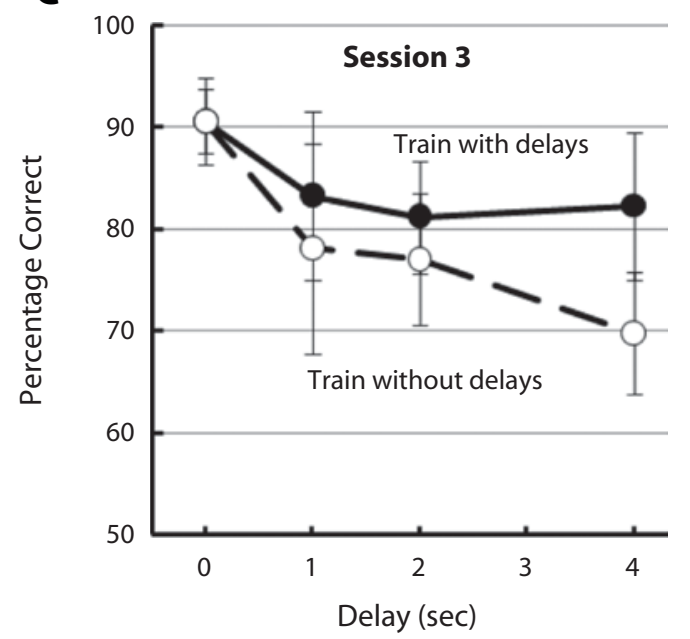

E

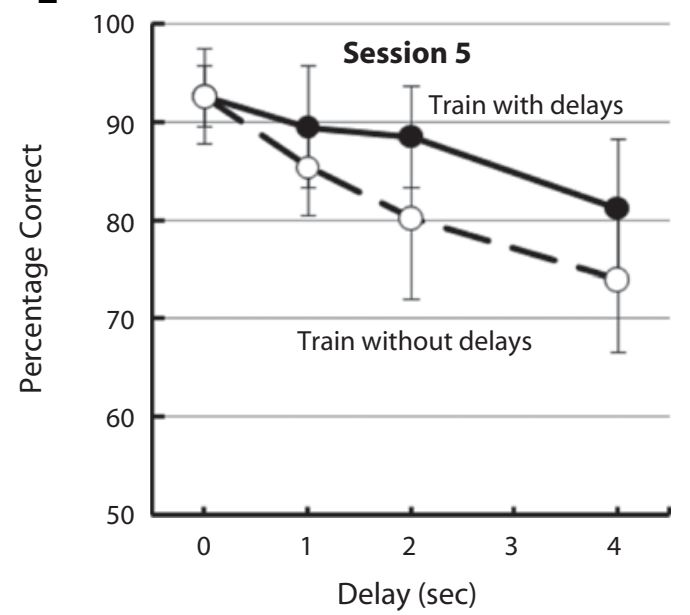

B

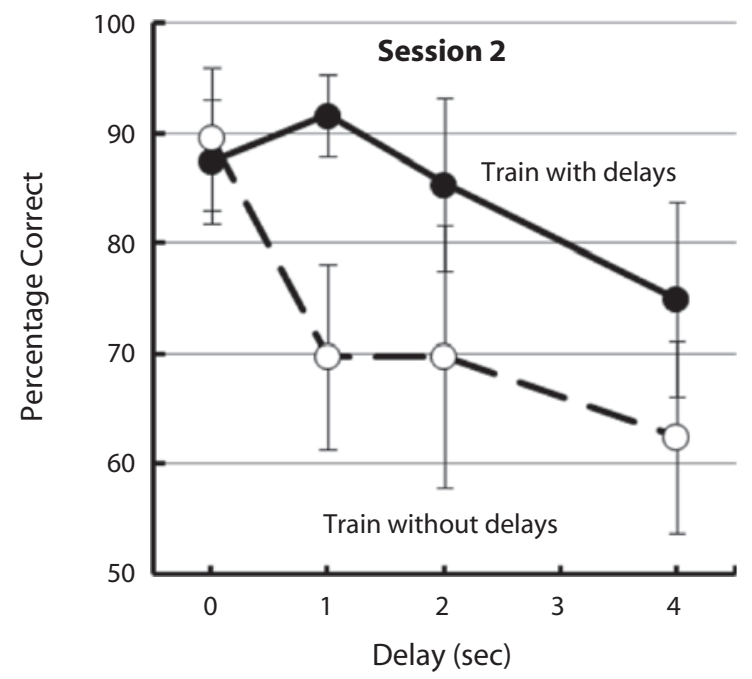

D

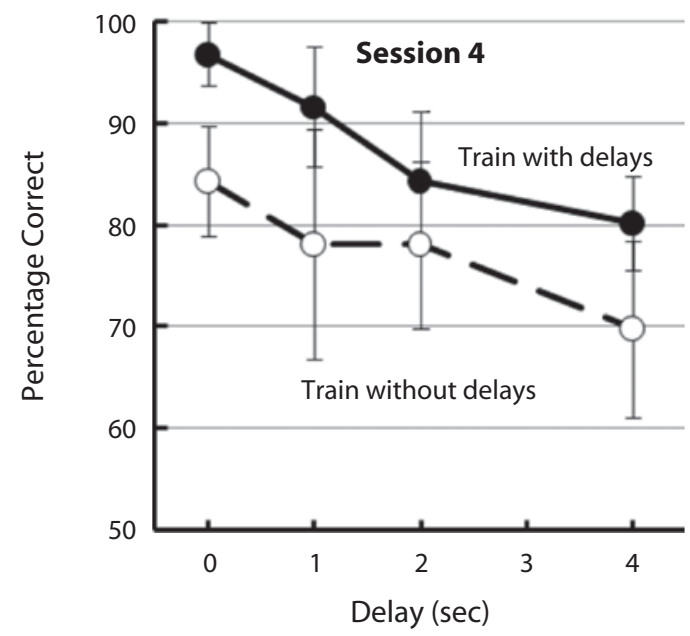

F

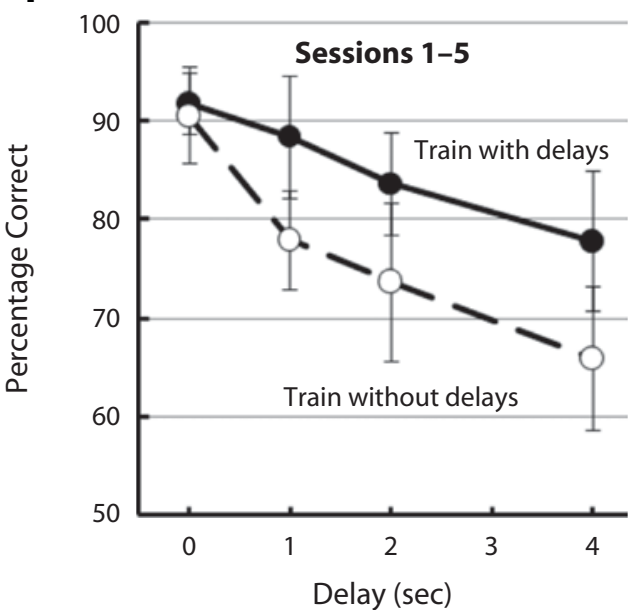

Figure 1. Matching accuracy as a function of the delay between the offset of the sample and the onset of the comparison stimuli for the pigeons in the experimental group, trained with delays of $0,1,2$, and $4 \mathrm{sec}$, and for those in the control group, trained with a 0 -sec delay, both tested with delays of $0,1,2$, and $4 \mathrm{sec}$. For the pigeons in the control group, testing consisted of the first five sessions after they had attained a criterion of two sessions at or above $87.5 \%$ correct on the 0 -sec delay trials. The pigeons in the experimental group were also trained to a criterion of two sessions at or above $87.5 \%$ correct on the 0 -sec delay trials, and the next five sessions were considered testing sessions, shown separately for each test session (A-E) and as the mean of the five test sessions (F). 


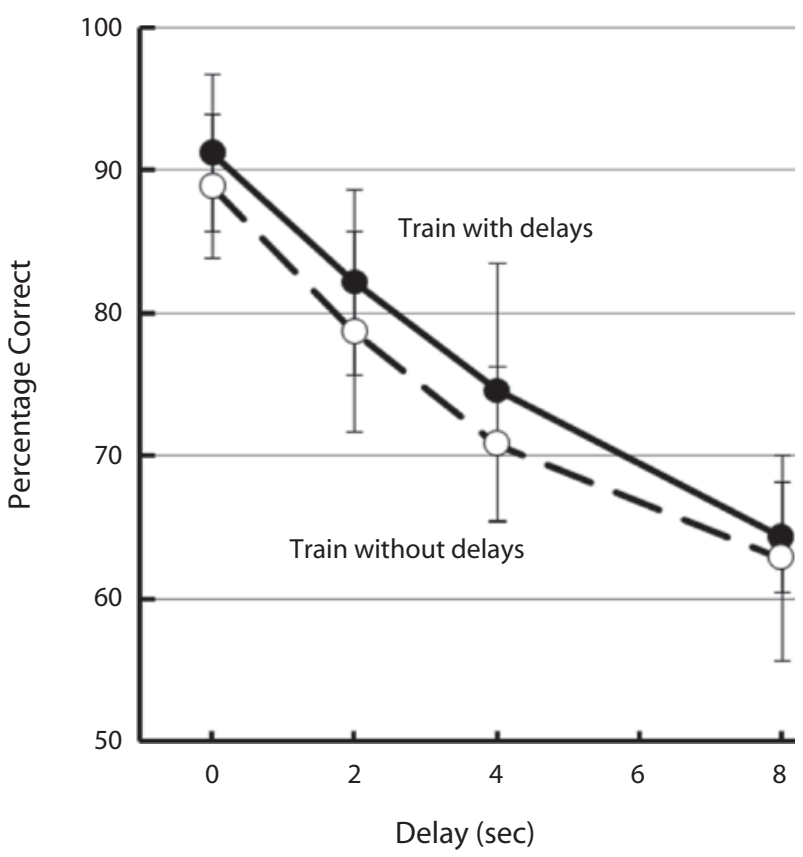

Figure 2. Matching accuracy, pooled over five sessions, as a function of delay $(0,2,4$, or $8 \mathrm{sec})$ between the offset of the sample and the onset of the comparison stimuli for the pigeons in the experimental group, trained with delays, and those in the control group, trained with a 0-sec delay, both originally tested with delays of $0,1,2$, and $4 \mathrm{sec}$.

responses at the 0 -sec delay and declined to only $81.5 \%$ correct at the 4-sec delay, a difference of only $6.4 \%$.

The present experiment involved only 15 test sessions and, although several delayed matching experiments have tested for more sessions (e.g., Grant, 1975, and Spetch \& Rusak, 1989, tested for 20 sessions, and Grant, 2000, tested for 60 sessions), others have not (e.g., Grant, 1981, tested for only 16 sessions; Santi \& Hope, 2001, tested for 15 sessions; Singer et al., 2006, tested for 10 sessions; and Clement \& Zentall, 2000, tested for only 1 session). But even in those studies in which there were more than 15 sessions, residual effects of differential experience may remain, and even if these residual effects are no longer present, the data have all been averaged for matching accuracy over all of the testing sessions, which would include the earlier sessions in which differential experience was likely to have played a role. Furthermore, many of the cited researchers tested with delays longer than those used in the present study, and longer delays would be even more likely to produce a larger generalization decrement.

One could argue that in the typical procedure with 0 -sec delayed matching training, although all of the nonzero delays are novel at the time of testing, they are equally novel, and one can thus obtain an unbiased estimate of the retention function by excluding matching accuracy at the 0 -sec delay. However, the degree of novelty of the delays, relative to the 0 -sec training value, should vary directly with their duration. That is, the longer the novel delay, the greater the difference from training and the greater the expected generalization decrement. Thus, variability in the similarity of the 0 -sec training delay to the longer delays is likely to differentially bias comparison of matching accuracy among the longer delays.

Another way to view the generalization decrement associated with novel sample-comparison delays is in terms of the expectation of the appearance of the comparison stimuli. If the animals expect the comparison stimuli to be presented immediately following the offset of the sample, the failure of their appearance at the expected time is likely to disrupt matching accuracy, and that disruption is likely to be proportional to the delay. This perspective can also account for the loss of matching accuracy when the animals are trained with a delay and the delay is shortened (see Sargisson \& White, 2001), because the unexpected premature appearance of the comparison stimuli is also likely to be disruptive of matching accuracy.

One might propose that in many experiments with delayed matching, it is the relative decline in matching accuracy with increasing delay as a function of condition, rather than the absolute decrement, that is of interest. For example, one might be interested in the relative effect on delayed matching accuracy of the number of pecks to the sample by the pigeons (Roberts, 1972). If so, the effect of the novelty of the delay (the generalization decrement) should be similar in the two conditions. But it is also possible that the generalization decrement and sample memory are not independent. In any event, the goal of a retention test should be to obtain a measure of the effect of a variable on memory, uninfluenced by a possible generalization decrement. Thus, one should avoid the potential contribution of a differential generalization decrement.

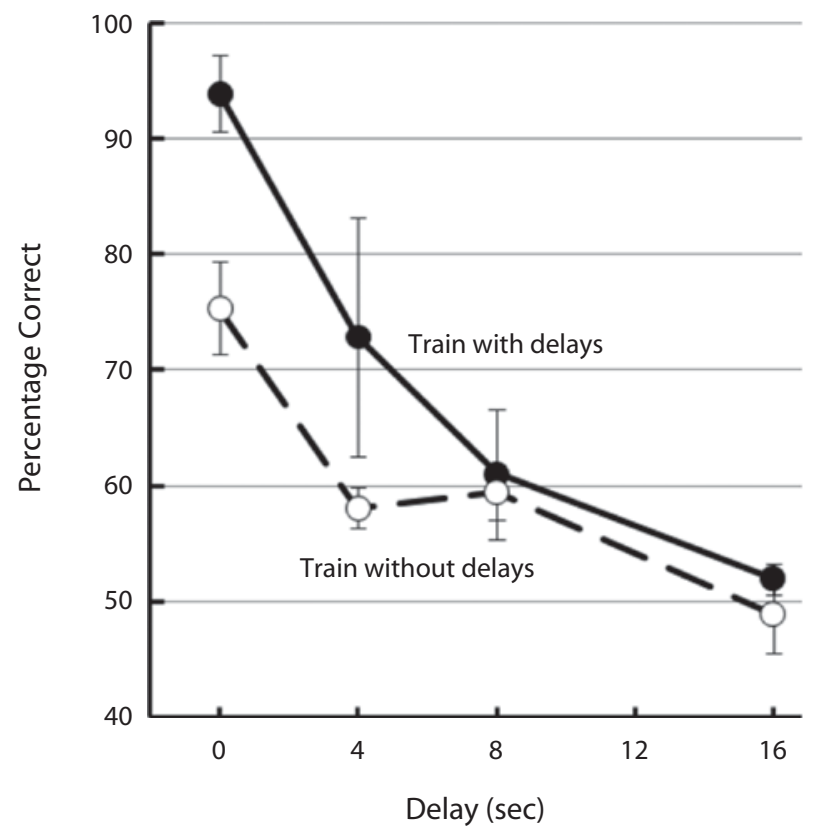

Figure 3. Matching accuracy, pooled over five sessions, as a function of delay $(0,4,8$, or $16 \mathrm{sec})$ between the offset of the sample and the onset of the comparison stimuli for the pigeons in the experimental group, trained with delays, and those in the control group, trained with a 0 -sec delay, both tested with delays of $0,1,2$, and 4 sec and then delays of $0,2,4$, and 8 sec. 
It could also be argued that all loss of matching accuracy with increasing delay is attributable to a change in temporal context (see, e.g., Bouton, Nelson, \& Rosas, 1999 ) and that it is not necessary to make a distinction between a generalization decrement and forgetting. On the other hand, the evidence that experience with delays tends to improve retention suggests that familiarity with delays (or the temporal expectation of the appearance of the comparison stimuli) plays a role in matching accuracy. Whether all forgetting results from the change in context or some forgetting can be attributed to memory loss, if one wants to obtain an unbiased estimate of retention, it is important that the generalization decrement attributable to the novelty of the delays be similar for all delays.

A second purpose of the present experiment was to ask whether extending the delays beyond those used in training for the experimental group would result in a discontinuous decrement in matching accuracy because of the added loss of control associated with the generalization decrement. Had there been an added decrement in matching accuracy attributable to the additional generalization decrement, one would have expected to see a sharper drop in matching accuracy from the familiar 4-sec delay to the unfamiliar 8 -sec delay, but the drop in matching accuracy appeared to be quite linear. Thus, within the limits of the delays tested (an increase from 4 to $8 \mathrm{sec}$ ), it does not appear that there was an additional generalization decrement.

The present research suggests that the standard procedure for assessing animal memory involving training to criterion on a conditional discrimination with no delay and only then introducing delays produces a bias due to the confound of memory loss with a generalization decrement. It is suggested that a less biased procedure should be used in memory research involving matching to sample (and likely other procedures as well) —one that involves equal training at all values of delay of interest and testing for memory loss with increasing delays only once the criterion performance is attained on the conditional discrimination without delays.

\section{AUTHOR NOTE}

This research was supported by National Institute of Mental Health Grant MH-063726 to T.R.Z. Correspondence concerning this article should be addressed to T. R. Zentall, Department of Psychology, University of Kentucky, Lexington, KY 40506 (e-mail: zentall@uky.edu).

\section{REFERENCES}

Berryman, R., Cumming, W. W., \& Nevin, J. A. (1963). Acquisition of delayed matching in the pigeon. Journal of the Experimental Analysis of Behavior, 6, 101-107.

Blough, D. S. (1959). Delayed matching in the pigeon. Journal of the Experimental Analysis of Behavior, 2, 151-160.

Bouton, M. E., Nelson, J. B., \& Rosas, J. M. (1999). Stimulus generalization, context change, and forgetting. Psychological Bulletin, 125, 171-186.

Clement, T. S., \& Zentall, T. R. (2000). Development of a singlecode/default coding strategy in pigeons. Psychological Science, 11, 261-264.

DeLong, R. E., \& Wasserman, E. A. (1981). Effects of differential reinforcement expectancies on successive matching-to-sample performance in pigeons. Journal of Experimental Psychology: Animal Behavior Processes, 7, 394-412.

Dorrance, B. R., Kaiser, D. H., \& Zentall, T. R. (2000). Event duration discrimination by pigeons: The choose-short effect may result from retention-test novelty. Animal Learning \& Behavior, 28, 344-353.

Eacott, M. J., Gaffan, D., \& Murray, E. A. (1994). Preserved recognition memory for small sets, and impaired stimulus identification for large sets, following rhinal cortex ablations in monkeys. European Journal of Neuroscience, 6, 1466-1478.

Grant, D. S. (1975). Proactive interference in pigeon short-term memory. Journal of Experimental Psychology: Animal Behavior Processes, 1, 207-220.

GRANT, D. S. (1976). Effect of sample presentation time on long-delay matching in the pigeon. Learning \& Motivation, 7, 580-590.

Grant, D. S. (1981). Stimulus control of information processing in pigeon short-term memory. Learning \& Motivation, 12, 19-39.

GRANT, D. S. (2000). Influence of intertrial interval duration on the intertrial agreement effect in delayed matching-to-sample with pigeons. Animal Learning \& Behavior, 28, 288-297.

Grant, D. S., \& RoberTS, W. A. (1973). Trace interaction in pigeons' short-term memory. Journal of Experimental Psychology, 101, 21-29.

RoberTs, W. A. (1972). Short-term memory in the pigeon: Effects of repetition and spacing. Journal of Experimental Psychology, 94, 74-83.

RoberTs, W. A. (1974). Spaced repetition facilitates short-term retention in the rat. Journal of Comparative \& Physiological Psychology, 86, 164-171.

Roberts, W. A. (1998). Principles of animal cognition. Boston: McGraw-Hill.

Roberts, W. A., \& Grant, D. S. (1976). Studies of short-term memory in the pigeon using the delayed matching-to-sample procedure. In D. L. Medin, W. A. Roberts, \& R. T. Davis (Eds.), Processes of animal memory (pp. 79-112). Hillsdale, NJ: Erlbaum.

SANTI, A., \& Hope, C. (2001). Errors in pigeons' memory for number of events. Animal Learning \& Behavior, 29, 208-220.

Santi, A., Lellwitz, J., \& Gagne, S. (2006). Pigeons' memory for sequences of light flashes: Reliance on temporal properties and evidence for delay interval/gap confusion. Behavioural Processes, 72, 128-138.

SARgisson, R. J., \& White, R. K. (2001). Generalization of delayed matching-to-sample performance following training at different delays. Journal of the Experimental Analysis of Behavior, 75, 1-14.

Sherburne, L. M., Zentall, T. R., \& Kaiser, D. H. (1998). Timing in pigeons: The choose-short effect may result from "confusion" between delay and intertrial intervals. Psychonomic Bulletin \& Review, 5, 516-522.

Singer, R. A., Klein, E. D., \& Zentall, T. R. (2006). The use of a single-code/default strategy by pigeons to acquire duration sample discriminations. Learning \& Behavior, 34, 340-347.

SPETCH, M. L., \& RUSAK, B. (1989). Pigeons' memory for event duration: Intertrial interval and delay effects. Animal Learning \& Behavior, 17, 147-156.

White, K. G. (1985). Characteristics of forgetting functions in delayed matching to sample. Journal of the Experimental Analysis of Behavior, 44, 15-34.

White, K. G. (2001). Forgetting functions. Animal Learning \& Behavior, 29, 193-207.

Zentall, T. R. (1997). Animal memory: The role of instructions. Learning \& Motivation, 28, 280-308.

Zentall, T. R., \& Hogan, D. E. (1977). Short-term proactive inhibition in the pigeon. Learning \& Motivation, 8, 367-386.

Zentall, T. R., Hogan, D. E., Howard, M. M., \& Moore, B. S. (1978). Delayed matching in the pigeon: Effect on performance of sample-specific observing responses and differential delay behavior. Learning \& Motivation, 9, 202-218.

(Manuscript received March 1, 2009; revision accepted for publication April 15, 2009.) 